\title{
Plasma motions in a short-lived filament related to a magnetic flux cancellation
}

\author{
F. Zuccarello ${ }^{1}$, V. Battiato ${ }^{2}$, L. Contarino ${ }^{1}$, P. Romano ${ }^{2}$, and D. Spadaro ${ }^{2}$ \\ 1 Dipartimento di Fisica e Astronomia - Sezione Astrofisica, Università di Catania, via S. Sofia 78, 95123 Catania, Italy \\ e-mail: fzu@oact.inaf.it \\ 2 INAF - Osservatorio Astrofisico di Catania, via S. Sofia 78, 95123 Catania, Italy
}

Received 12 October 2006 / Accepted 2 February 2007

\section{ABSTRACT}

\begin{abstract}
Context. In recent years the mechanisms responsible for filament formation and evolution have been investigated by many authors. In particular, the role played by the processes of magnetic flux cancellation in building up or destroying filaments is still a matter of debate.

Aims. In this paper we analyze the evolution of an active region filament that formed in NOAA 10407 on 14 July 2003 , to investigate the phenomena responsible for its destabilization and short lifetime $(\sim 12 \mathrm{~h})$.

Methods. This analysis is based on high-resolution $\mathrm{H}_{\alpha}$ data acquired by THEMIS operating in IPM mode, on $\mathrm{H}_{\alpha}$ data acquired at Big Bear Solar Observatory, and on MDI/SOHO magnetograms. Using these data, we determined the morphological, dynamical, and magnetic evolution of the filament.

Results. The chromospheric images show two dark surges occurring sequentially in the northern part of the filament, besides two bright $\mathrm{H}_{\alpha}$ patches located in the same area; from analysis of the photospheric magnetograms, we could infer that a magnetic flux cancellation had occurred in this area.

Conclusions. The presence of a cancelling magnetic feature (CMF) in the same area where the dark $\mathrm{H}_{\alpha}$ surges occurred, the temporal behavior of the velocity fields in the surges, and the presence of bright $\mathrm{H}_{\alpha}$ patches in the CMF area, suggest a scenario where the coronal arcade initially sustaining the filament might have undergone consecutive reconnection processes. From the concurrence of these events with the filament activation and successive disappearance, we believe that the arcade field lines, after the reconnection events, changed such that the plasma filament was no longer confined in the arcade: this led to its destabilization and disappearance.
\end{abstract}

Key words. Sun: activity - Sun: filaments - Sun: magnetic fields

\section{Introduction}

Solar filaments are optimally seen in the $\mathrm{H}_{\alpha}$ line, where they appear as dark, narrow $\left(\sim 10^{3}-10^{4} \mathrm{~km}\right)$, and often long $\left(\sim 10^{5} \mathrm{~km}\right)$ structures. On the other hand, in coronal images, the existence of a filament can also be inferred by the presence of a so-called EUV filament channel (Schwartz et al. 2004).

Even though the basic theory of the formation of filaments, via condensation of hot coronal plasma or injection of material from the cool chromosphere (e.g., Malherbe 1989; Tandberg-Hanssen 1995), is understood fairly well, application to actual observations is still problematic. A recent review by Martin (1998) summarizes important progress in the understanding of filament formation from an observational point of view. However, all the conclusions were drawn from observations of normally developed filaments, i.e., filaments that self-generated without being catalyzed by particular exterior agents, such as those proposed by, e.g., Martens \& Zwaan (2001), Chae (2003), and Lin et al. (2005).

Martens \& Zwaan (2001), for instance, present a model of the origin and evolution of filaments, based on the convergence and cancellation of the photospheric magnetic field (see also Amari et al. 2003). Along the same line of research, Chae et al. (2001) analyzed MDI magnetograms and conclude that the formation of a filament in an active region (NOAA 8668) is the visible manifestation of the creation of a large magnetic structure that consists of a flux rope and an overlying sheared arcade, due to a sequence of magnetic flux cancellation events.

Moreover, several authors have stressed that the process of magnetic flux cancellation can also play an important role in filament destabilization and eruption (Wang et al. 1996; Kim et al. 2001; Martens \& Zwaan 2001; Amari et al. 2003). In particular, Contarino et al. (2003), from the comparison of high-resolution $\mathrm{H}_{\alpha}$ observations with MDI magnetograms, were able to infer that a filament observed in NOAA 9445 underwent an initial phase of bifurcation and a successive partial eruption, due to a cancelling magnetic feature occurring close to the site of bifurcation.

In this framework, we recall that there are several observational signatures of magnetic-flux cancellation: decrease in magnetic flux, converging motions of opposite polarities, brightening in chromospheric and coronal lines, and plasma motions like surges and jets. Specifically, surges are collimated ejections of chromospheric matter into coronal heights. They can be seen as dark features in $\mathrm{H}_{\alpha}$ images moving upward at $50-200 \mathrm{~km} \mathrm{~s}^{-1}$, reaching heights of up to $200 \mathrm{Mm}$ and typically lasting for 10-20 min. The ejected matter fades or returns into the chromosphere apparently along the trajectory of ascent (Svestka 1976; Tandberg-Hanssen 1977; Schmieder et al. 1994). Sometimes surges evolve over a small dark mound-like region forming an inverted Y configuration. Kurokawa \& Kawai (1993) suggest that this shape can be explained by magnetic reconnection between an emerging flux and a preexisting surrounding region. Moreover, Chae et al. (1999) and Zhang et al. (2000) 
studied surges in emerging flux regions (EFRs), and find magnetic cancellations between the preexisting field and the newly emerging one at the footpoints of the surges. Such observations provide additional evidence supporting reconnection, and several reconnection models have already been proposed (Canfield et al. 1996; Yokohawa \& Shibata 1996).

In this paper we present the observations of an active region filament formed in NOAA 10407 on 14 July 2003 and characterized by an average length of $\sim 20$ arcsec and a lifetime of $\sim 12 \mathrm{~h}$. The filament was characterized by a very dynamic evolution and by the presence of two consecutive surge events. We show that these dark surges were related to a process of magnetic flux cancellation occurring in the magnetic area containing the filament, and we also discuss the consequences of these phenomena on the filament stability.

\section{Observations and description of NOAA 10407}

We have studied active region NOAA 10407 using data acquired by THEMIS/IPM (see Cavallini 1998, for a description of the instrument), BBSO $\mathrm{H}_{\alpha}$ telescope, and MDI/SOHO. This active region appeared on 11 July and vanished on 17 July 2003. It scarcely developed (max longitudinal extension $\sim 70$ arcsec), exhibiting the formation of a single spot, several pores, and some filaments. During its lifetime it did not show any flare activity. We refer the reader to Zuccarello et al. (2005) for a more detailed description of the observations and of the active region's evolution.

Figure 1a depicts a composition of $\mathrm{H}_{\alpha}$ images of NOAA 10407 acquired on 14 July 2003 at 9:05 UT by THEMIS/IPM (the instrument acquired images of two sectors with a field of view of $24 \times 24 \mathrm{Mm}^{2}$, almost aligned along the East-West direction and overlapped by $\sim 10$ arcsec). The time interval covered by THEMIS ranges between 8:59 UT and 9:51 UT; therefore, in order to investigate the evolution of the active region, we also used $\mathrm{H}_{\alpha}$ images acquired at BBSO in the following hours.

BBSO high-resolution $\mathrm{H}_{\alpha}$ images were acquired from 17:35 to $23: 24 \mathrm{UT}$, using the $65 \mathrm{~cm}$ reflector equipped with a Zeiss $\mathrm{H}_{\alpha}$ filter of $0.25 \AA$ bandwidth. The cadence between two consecutive images acquired at the same wavelength is $\sim 90 \mathrm{~s}$ with 0.3 arcsec pixel resolution. Figure $1 \mathrm{~b}$ shows a sample of a highresolution $\mathrm{H}_{\alpha}$ image acquired at 23:14 UT on 14 July at BBSO.

To study the magnetic field configuration and evolution of NOAA 10407 , we used full-disc magnetograms acquired by MDI/SOHO (Scherrer et al. 1995) between 00:03 UT and 16:03 UT, with an average time cadence of $96 \mathrm{~min}$ and a spatial resolution of 4 arcsec. To determine the details of the magnetic configuration, we also used high-resolution magnetograms acquired by MDI at 02:34 UT, 10:34 UT (see Fig. 1c), 22:34 UT.

The THEMIS image acquired in the center of the $\mathrm{H}_{\alpha}$ line shows an arch-filament system composed of $\sim 10$ arch filaments (AF), approximately parallel and located across the main polarity inversion line (PIL). These AFs have several lengths, up to a maximum of $\sim 15 \mathrm{Mm}$, and appear to connect the pores of opposite polarities. Their morphology and dynamics has been described in Zuccarello et al. (2005). In the North-West area, a small sunspot $(d \sim 6 \mathrm{Mm})$ is visible.

This $\mathrm{H}_{\alpha}$ image also exhibits a small filament (length $\sim 14 \mathrm{Mm}$ ), located South-East of the spot and oriented in the North-South direction (see the arrow in Fig. 1a). Close to the Northern part of the filament, we also observe a bright $\mathrm{H}_{\alpha}$ patch $(d \sim 1.5 \mathrm{Mm})$. The filament was located near the center of the solar disc (W05 N10) and observed on the solar

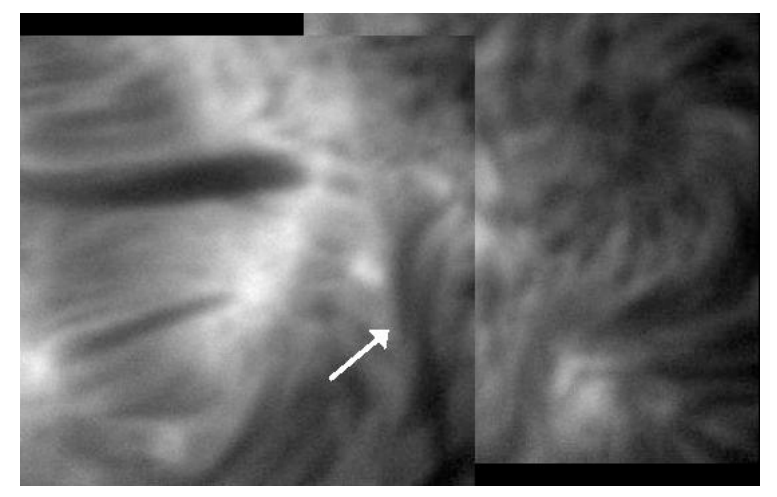

(a)

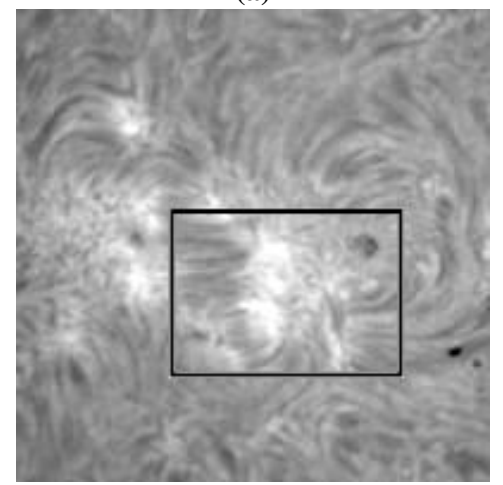

(b)

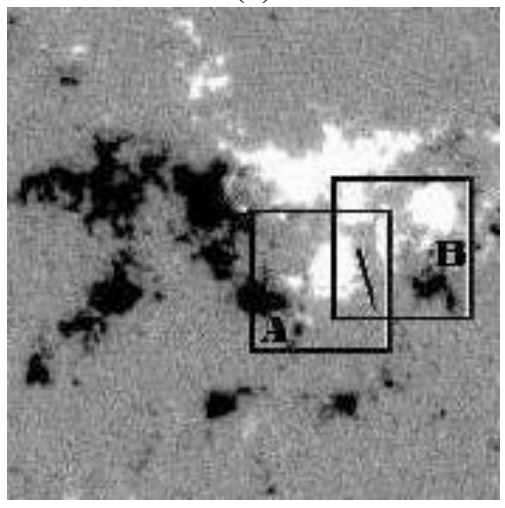

(c)

Fig. 1. a) Reconstruction of NOAA 10407 obtained from two images acquired by THEMIS in the center of the $\mathrm{H}_{\alpha}$ line, on 14 July 2003 at 9:05 UT. The white arrow indicates the location of the filament investigated. The fov is $\sim 40 \times 28 \mathrm{Mm}^{2}$; b) BBSO high-resolution $\mathrm{H}_{\alpha}$ image acquired at 17:35 UT (fov $\sim 90 \times 90 \mathrm{Mm}^{2}$ ); the rectangle indicates the field of view correspondent to Fig. 1a; c) high-resolution magnetogram acquired by MDI at 10:34 UT with fov of $\sim 90 \times 90 \mathrm{Mm}^{2}$. The black line indicates the position of the filament and the rectangles indicate the two fields of view scanned by the THEMIS telescope. In the images North is on the top, West to the right.

surface for no longer than $12 \mathrm{~h}$. In the BBSO image shown in Fig. 1b, the filament is no longer visible.

In Fig. 1c the filament is overlaid on the corresponding MDI magnetogram, and a line marks its orientation (the location of the filament has been corrected for the time difference between the magnetogram and the $\mathrm{H}_{\alpha}$ image).

\section{Data analysis}

For the analysis of chromospheric data we corrected the THEMIS/IPM data by applying the standard dark current and flat field corrections (e.g., Contarino et al. 2003). The method used to reconstruct the profile of the $\mathrm{H}_{\alpha}$ line, as well as the one 

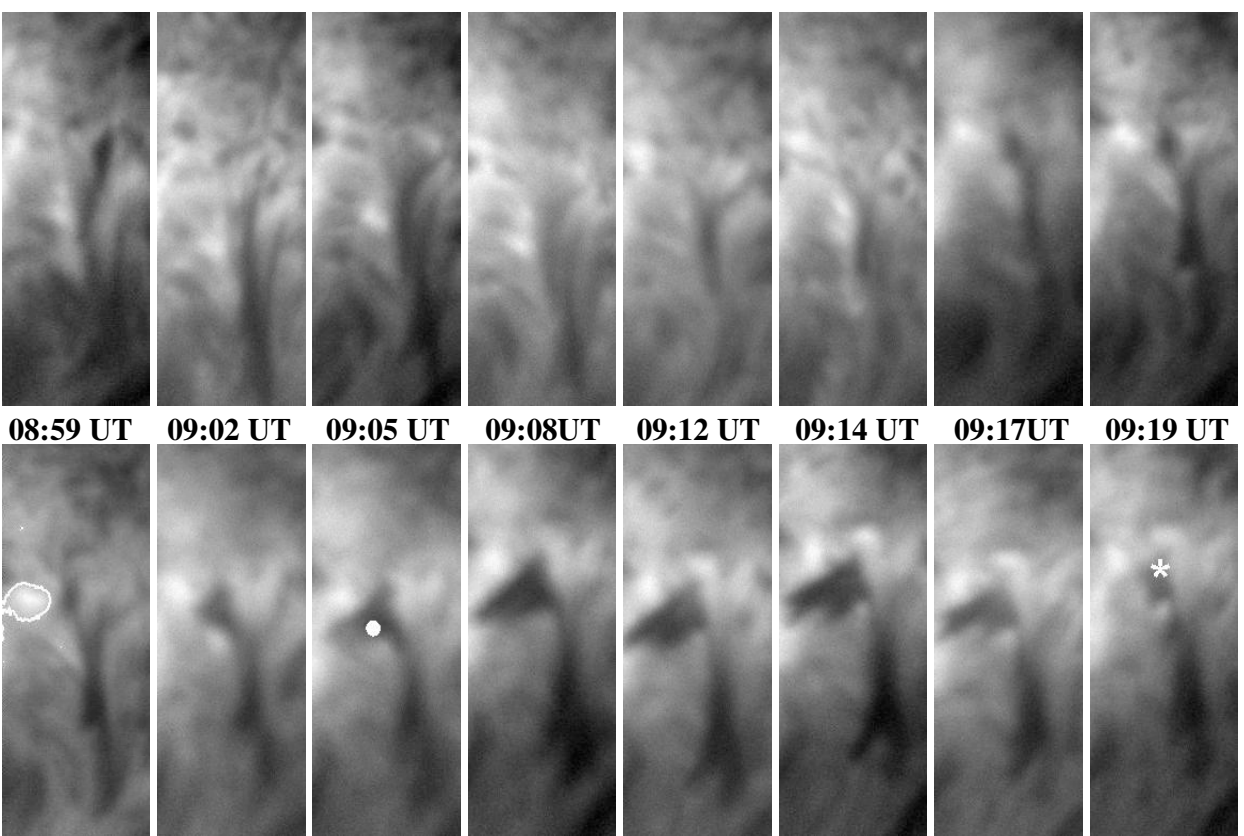

09:08UT
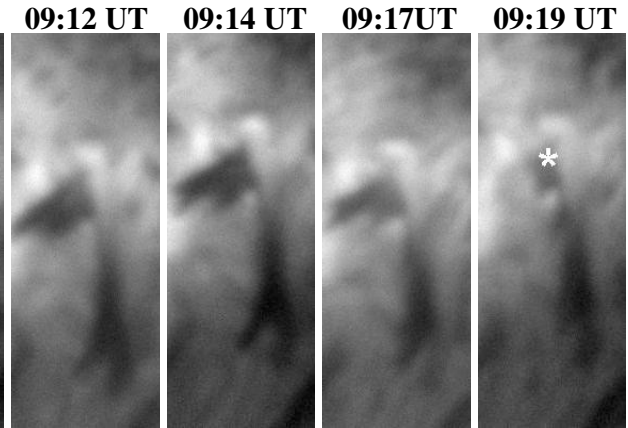

09:20 UT

09:22 UT

09:24 UT

09:25UT

09:26 UT

09:27 UT

09:28UT

09:29 UT
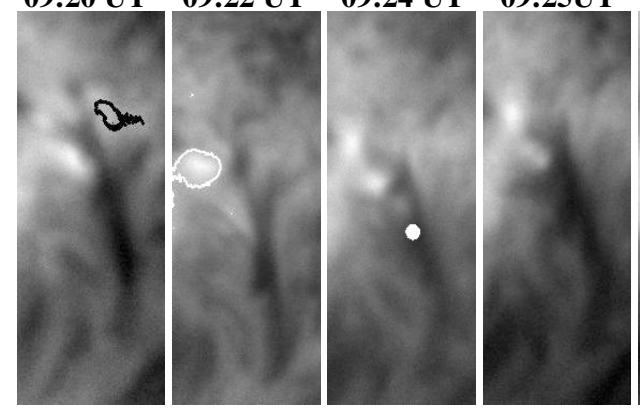

09:31 UT

09:32 UT

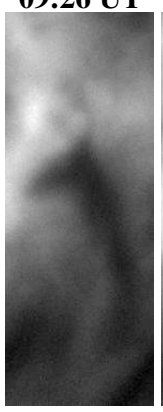

09:37 UT

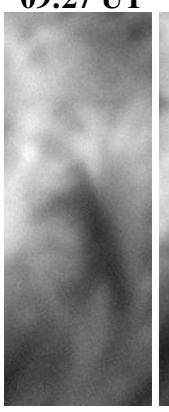

09:38 UT
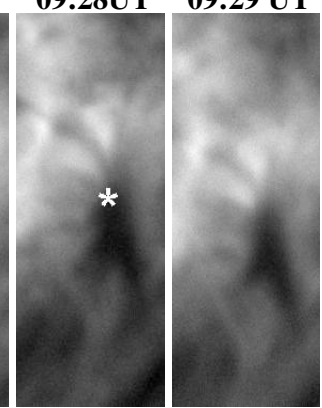

09:39UT 09:40 UT

Fig. 2. Evolution of the filament in the center of the $\mathrm{H}_{\alpha}$ line during the time interval 8:59-9:40 UT. The images are not always aligned along the vertical direction due to the different fields of view scanned by THEMIS. The circle (asterisk) indicates the point of the maximum upward (downward) velocity at the times when it peaks (see the plots in Fig. 4a). The white (black) contours in the images acquired at 09:20 and 09:32 UT (09:31 UT) indicate a bright patch associated with an upward (downward) motion. The fov is $\sim 10 \times 24 \mathrm{Mm}^{2}$.

used to determine the velocity along the line of sight (los), are described in Spadaro et al. (2000) and Zuccarello et al. (2005). The estimated error in the los velocity is $\pm 1 \mathrm{~km} \mathrm{~s}^{-1}$.

To determine the magnetic field configuration and evolution of the filament investigated, we selected one MDI full disc magnetogram every 96 min, from 00:03 to 16:03 UT on 14 July 2003. We also utilized MDI and BBSO high-resolution magnetograms to study the details of the photospheric magnetic field's evolution near the filament.

Moreover, to determine the evolution of the magnetic flux in the active region, we used a collection of IDL programs, YAFTA (Yet Another Feature Tracking Algorithm; Welsh \& Longcope 2003). YAFTA identifies flux elements in a high-resolution magnetogram and matches these elements between consecutive magnetograms. Then we analyzed the YAFTA results to verify the presence of magnetic flux cancellation events and to estimate the cancelled flux.

\section{Results}

Figure 2 shows the filament observed by THEMIS in the $\mathrm{H}_{\alpha}$ line center between 8:59 and 9:40 UT. We can see that this structure is characterized by a very dynamic evolution and by some features appearing as dark $\mathrm{H}_{\alpha}$ surges. They have a typical size of $\sim 8 \mathrm{Mm}$ and a lifetime of 10-15 $\mathrm{min}$. The first surge becomes visible at 9:20 UT. Its size increases rapidly with time, reaching the maximum around 9:26 UT, and then begins to fade at its base till 9:31 UT, when the surge material is not visible anymore. The second surge reaches its maximum extension at 09:37 UT and displays a similar behavior, but is smaller and not as dark.

Figure 3 shows some los velocity maps obtained from the analysis of the $\mathrm{H}_{\alpha}$ profile in the filament area. We plot the velocity contours every $3 \mathrm{~km} \mathrm{~s}^{-1}$ over the image taken in the center of the $\mathrm{H}_{\alpha}$ line. Thick and thin contours indicate positive (upward) and negative (downward) los plasma motion, respectively. The maps show that the velocity in the East-Southern part of the filament is generally upward, with values about $4 \mathrm{~km} \mathrm{~s}^{-1}$. Contemporaneously, the northern part of the filament exhibits negative velocities $\left(\sim 5 \mathrm{~km} \mathrm{~s}^{-1}\right)$.

During the time intervals 9:20-9:26 UT and 9:32-9:36 UT, simultaneous flows, in opposite directions, are seen close to the northern part of the filament. More precisely, at 09:20 UT, when the first surge appears, the small dark jet (with a flag shape) exhibits an upward velocity; this direction of motion is evident until 09:26 UT, when the small flag, reaching the maximum extension, is characterized by a downward motion (until 09:30 UT). When the second surge starts (i.e., at 


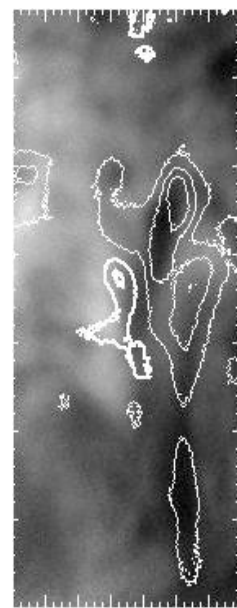

08:59

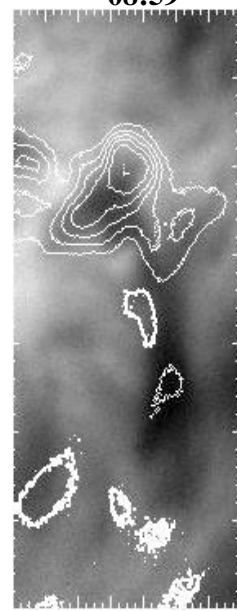

09:29

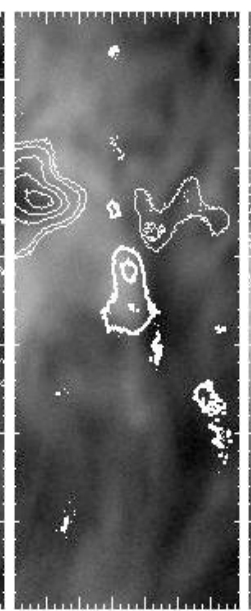

09:15

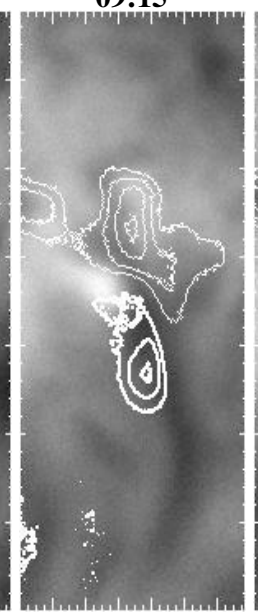

09:32

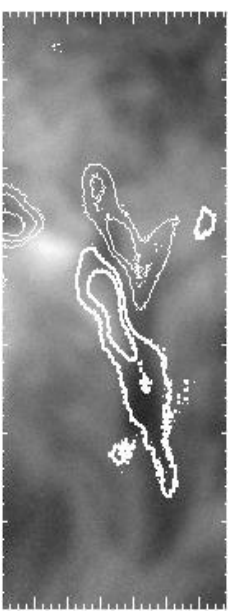

09:20

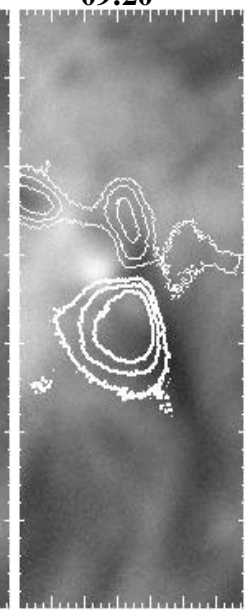

09:34

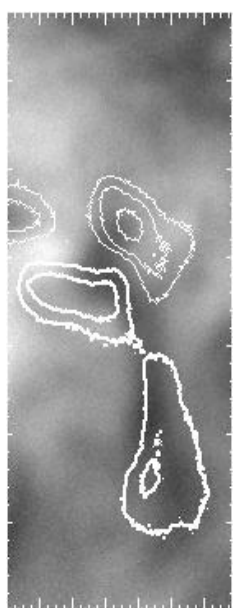

09:24

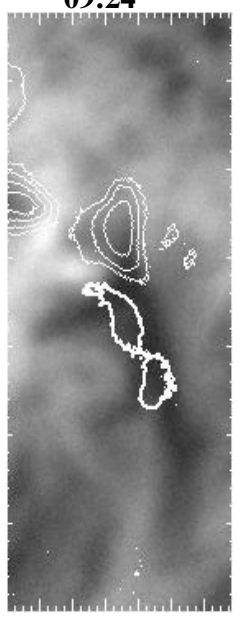

09:36

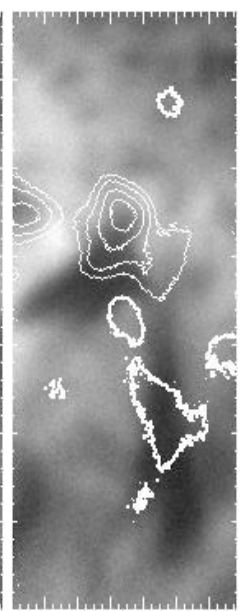

09:26

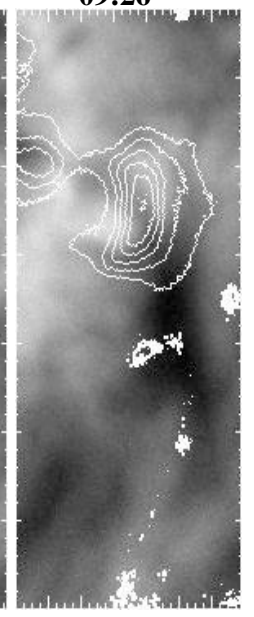

09:39

Fig. 3. Velocity maps obtained using the $\mathrm{H}_{\alpha}$ profile. Each map is plotted over an image acquired in the center of the $\mathrm{H}_{\alpha}$ line. Thick (thin) contours indicate upward (downward) plasma motions. The velocity contours are drawn every $3 \mathrm{~km} \mathrm{~s}^{-1}$. The fov is $\sim 10 \times 24 \mathrm{Mm}^{2}$.

09:32 UT), we again observe an initial upward motion within a few minutes followed by a downward motion.

Moreover, during the surge events, two $\mathrm{H}_{\alpha}$ bright patches were observed near the flag: the eastern patch showed an upward motion, while the western patch was characterized by a downward motion.

Figure 4a reports the maximum los upward velocities and maximum downward velocities measured in the filament, as a function of time (the vertical lines mark the time intervals of the occurrence of the two surges). We can see that both upward and downward motions were present in the filament area during the time interval analyzed. Moreover, where the surges occur, the downward velocity peaks with a delay of about 5 min with respect to the upward velocity.

In Fig. 4b we report the variation of the filament area as a function of time. (The filament area is estimated considering the number of pixels with an intensity lower than $600 \mathrm{DN} / \mathrm{px}$, which is the threshold value adopted to define the contour of the filament.) We can see that there is a decrease before the first surge and then a slow increase in the filament area; during the first surge the area increases steeply and there is a successively weaker, but still evident, increase during the second surge.

Figure $4 \mathrm{c}$ shows the variation in the intensity in the $\mathrm{H}_{\alpha}$ bright patch close to the flag area, associated with the upflow motion (see also Fig. 2): the increase in intensity is strongly correlated with the two surge events, and the peak is reached, in both cases, at the beginning of the surges. Figure $4 d$ then shows the intensity variation in the bright patch associated with the downward motion: in this case, the maximum intensity is recorded after the surges, i.e. when the plasma fell down to the bottom of the chromosphere.

Figure 5 shows the evolution of the magnetic flux distribution in NOAA 10407 on July 14. The filament investigated formed along the polarity-reversal boundary between some pores of positive polarity and a small negative polarity (see also Fig. 1c). We note that the negative magnetic flux was cancelled and eventually replenished by a newly emerging flux of opposite polarity. In order to prove this behavior, we show in Fig. 6 the variation in the negative (Fig. 6a) and positive (Fig. 6b) magnetic flux in the area enclosed by the white and black contours in Fig. 5 (06:23 UT), respectively. The averaged area enclosed in the black contour is $220 \mathrm{Mm}^{2}$. We can see that, during the time interval 01:39-12:47 UT, the negative magnetic flux shows an initial increase up to a maximum registered at 06:23 UT; successively the negative flux decreases, until it becomes negligible (12:47 UT). During the same time interval, the positive magnetic flux shows a continuous increase.

Therefore, while this area was initially characterized by a phase of magnetic flux emergence (increase in both negative and positive flux until $\sim 08: 00 \mathrm{UT}$ ), a process of magnetic flux 


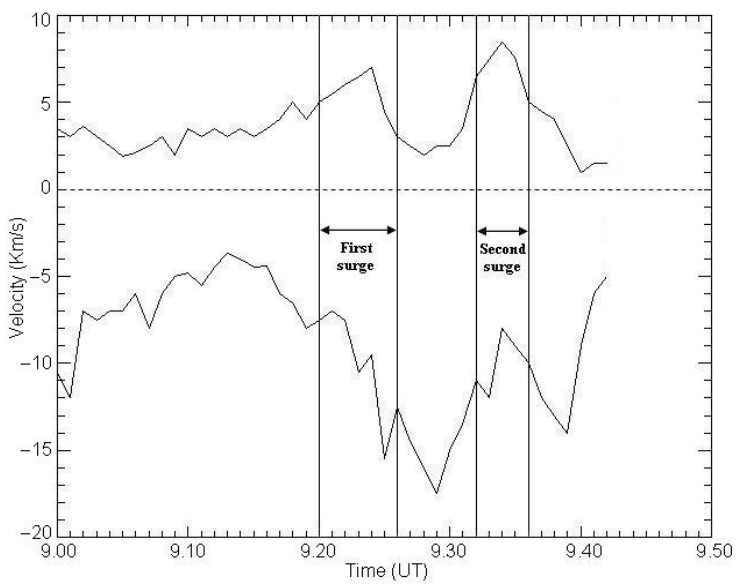

(a)

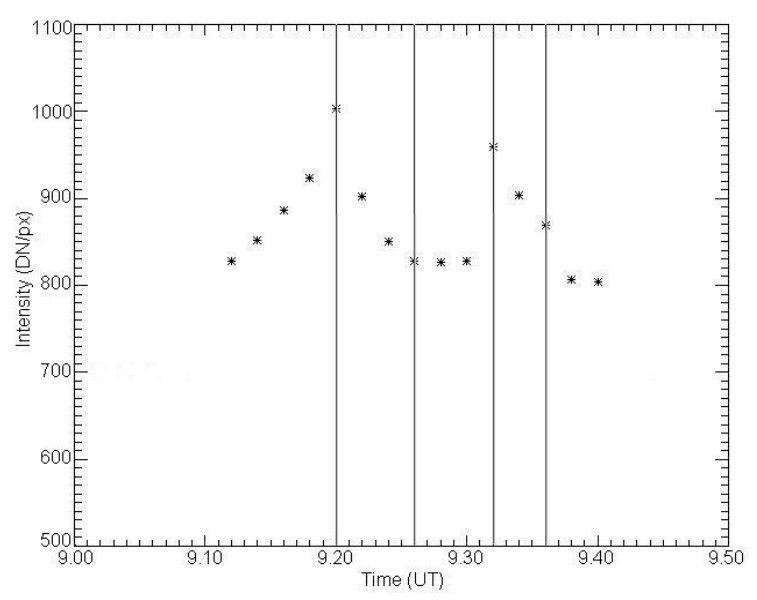

(c)

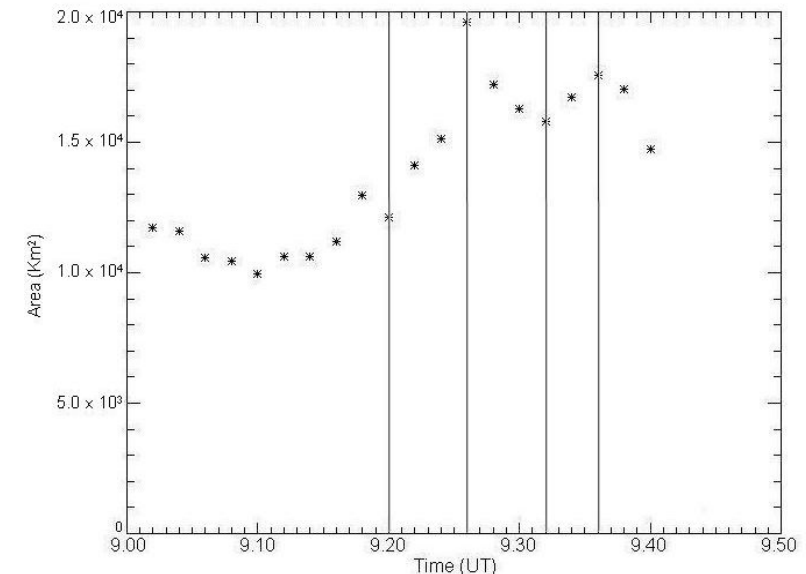

(b)

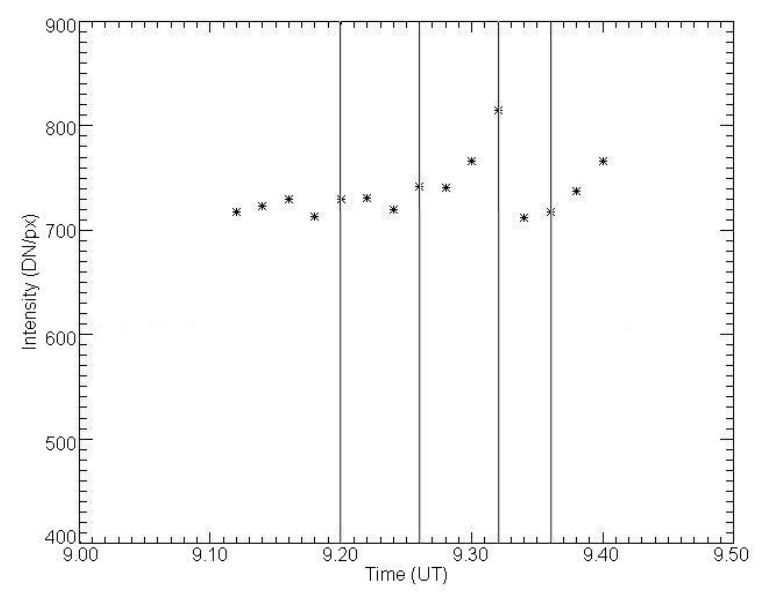

(d)

Fig. 4. a) Maximum values of upward and downward velocities in the filament, as a function of time; b) area characterized by the same level of darkness in the filament, as a function of time; c) intensity in the patch associated with the upflow motion, as a function of time; d) intensity in the patch associated with the downflow motion, as a function of time.

cancellation might explain the negative polarity decrease from 08:00 to 12:47 UT. On the other hand, we stress that the order of magnitude of the two fluxes $\left(\sim 3.0 \times 10^{18} \mathrm{Mx}\right.$ for the negative knot and $1.5 \times 10^{20} \mathrm{Mx}$ for the positive area) is such that the cancellation of the corresponding positive counterpart cannot be disentangled within the general increasing trend of the positive flux. On the basis of this interpretation, we conclude that a cancelling magnetic feature $(\mathrm{CMF})$ occurred between the two opposite polarities enclosed in the contours in Fig. 5.

Using YAFTA, we determined the amount of negative magnetic flux lost by cancellation in the CMF, and obtained a value of $1.2 \times 10^{18} \mathrm{Mx}$ in the time interval 02:34-10:34 UT (corresponding to a cancelling rate of $1.5 \times 10^{17} \mathrm{Mx} / \mathrm{h}$ or $4.2 \times$ $\left.10^{13} \mathrm{Mx} / \mathrm{s}\right)$. By comparing the THEMIS $\mathrm{H}_{\alpha}$ images and the magnetograms, we could infer that the region characterized by the presence of the CMF was cospatial with the northern part of the filament and with the bright $\mathrm{H}_{\alpha}$ patch associated to the upward motion (Fig. 2, image at 9:20 UT).

\section{Discussion and conclusions}

Using IPM/THEMIS images, and BBSO and MDI/SOHO magnetograms, we have investigated the behaviour of the chromospheric features and of the photospheric magnetic field in active region NOAA 10407 during the evolution of a short-lived filament: the results obtained indicate that the phenomena occurring in the filament were associated to a process of magnetic flux cancellation.

We observed in fact some signatures indicating the occurrence of a cancelling magnetic feature that was due to the interaction between a low negative magnetic flux concentration, already existing at the beginning of the observations, and an emerging positive one. These signatures were: (1) decrease in the negative magnetic flux (we determined the amount of magnetic flux lost by cancellation in the CMF: $1.2 \times 10^{18} \mathrm{Mx}$ ); (2) brightening in chromospheric lines, where the analysis of the THEMIS images showed that an $\mathrm{H}_{\alpha}$ emission patch appeared near the northern end of the filament (see the sequence in Fig. 2) and that this bright patch was generally characterized by an upward motion; (3) plasma motions like jets and surges. In the same filament end where the bright $\mathrm{H}_{\alpha}$ patch was observed, two consecutive $\mathrm{H}_{\alpha}$ dark surges were observed during the time interval 09:20-09:38 UT. Studying the velocity fields associated with the $\mathrm{H}_{\alpha}$ dark surges indicated initial upward motions (maximum velocity $\sim 8 \mathrm{~km} \mathrm{~s}^{-1}$ ), followed within 3-5 min by downward motions characterized by higher velocity values $\left(\sim 20 \mathrm{~km} \mathrm{~s}^{-1}\right)$ (see Fig. 4a). 

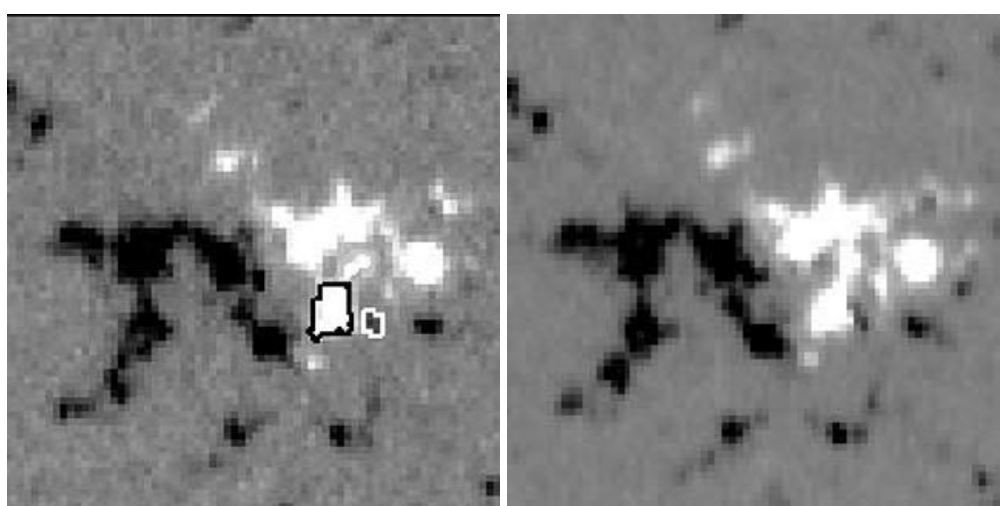

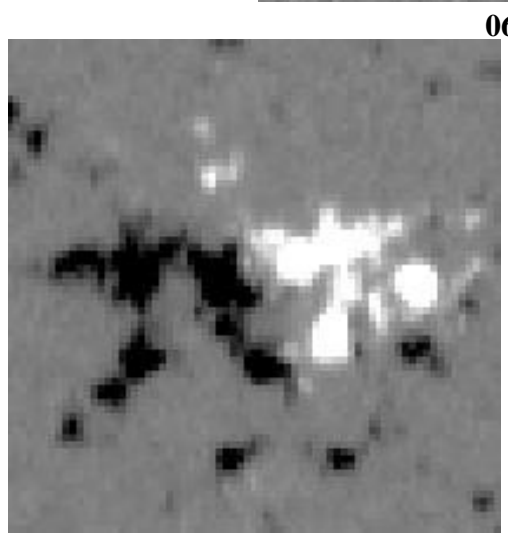

09:39

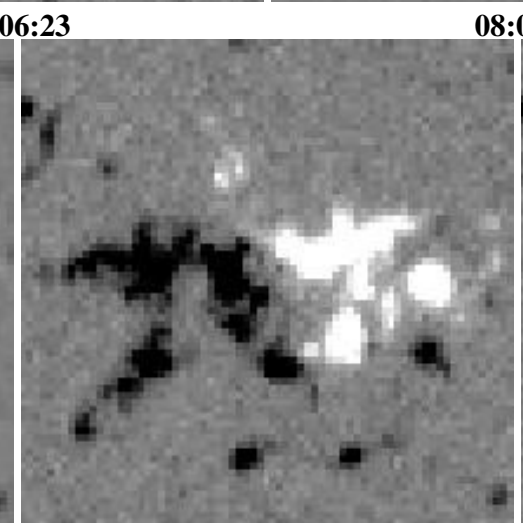

11:11

08:03

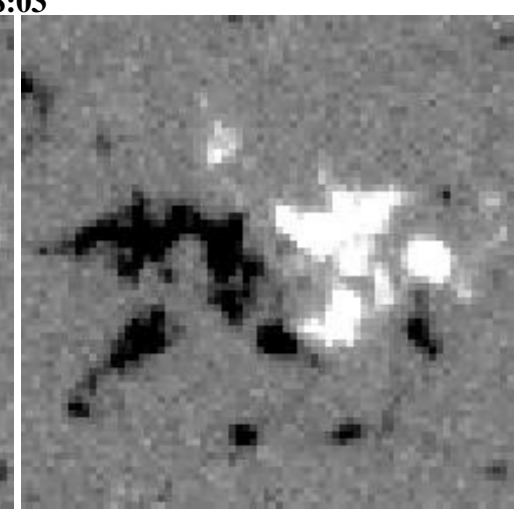

12:47

Fig. 5. MDI/SOHO (06:23-12:47 UT) low-resolution magnetograms showing the presence of the CMF in the active region. The black (white) contour in the magnetogram acquired at 6:23 UT indicates the area used to evaluate the positive (negative) flux variation. The field of view is $\sim 95 \times 95 \mathrm{Mm}^{2}$.

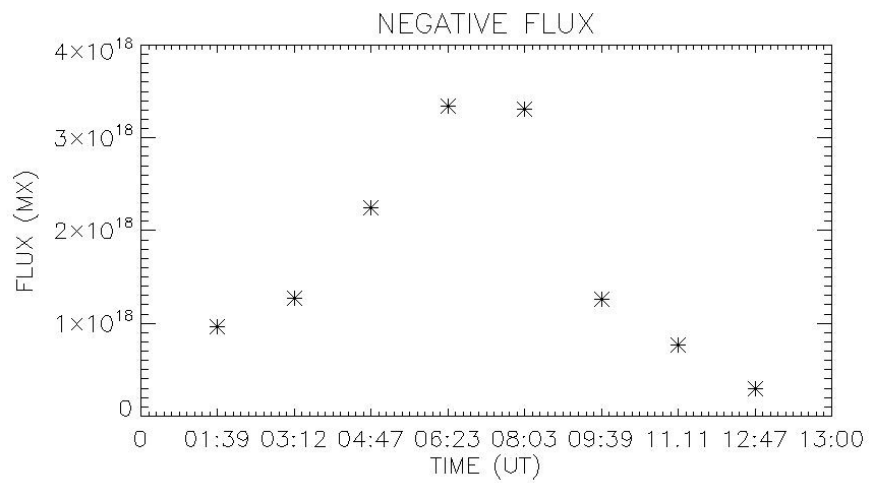

(a)

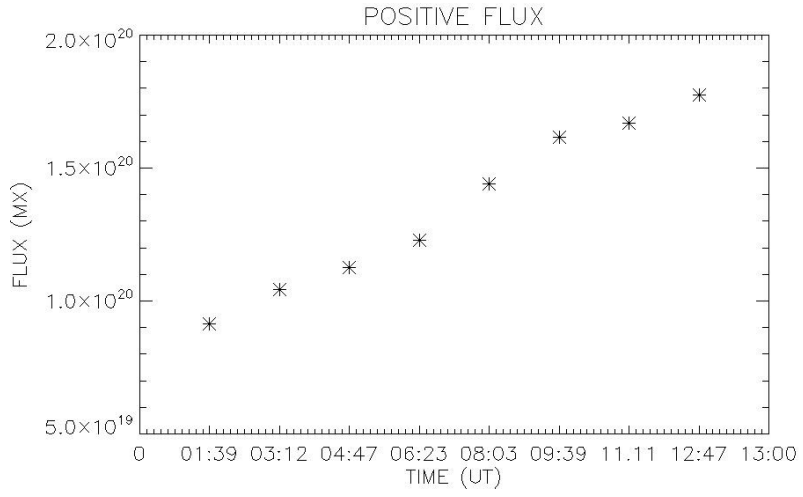

(b)

Fig. 6. Variation in the negative a) and positive b) flux in the CMF, deduced from low-resolution MDI magnetograms.

The comparison between the $\mathrm{H}_{\alpha}$ images and the MDI magnetograms showed that these surges were associated, both spatially and temporarily, with the sites of magnetic flux cancellation. This indicates that the surges may have resulted from the process of magnetic reconnection occurring in the low atmosphere when the negative magnetic fragment cancelled with the positive emerging flux. In this context, it is worthwhile to stress the importance of flux cancellation in the acceleration mechanisms of $\mathrm{H}_{\alpha}$ surges: Yokoyama \& Shibata (1996) successfully show that both hot plasma and cool plasma can be accelerated by magnetic reconnection driven by emerging flux. According to their model, $\mathrm{H}_{\alpha}$ surges are cool jets that are ejected by the sling-shot effect due to reconnection.
Another interesting result stems from the analysis of the filament area vs. time (Fig. 4b): it appeared that coinciding with the surge activity, the filament area increased and later on decreased.

We interpret these phenomena as due to the filament being replenished with plasma because of reconnection occurring between the magnetic field lines supporting it and those emerging from subphotospheric layers. In this scenario, the surges we observed witness the plasma jets that might be associated with the reconnection occurring during the magnetic flux cancellation. Moreover, a first phase of blueshift and a successive phase of redshift indicate that the plasma was ejected toward the external layers when the surge started, and later on it was directed toward the chromosphere basis when it fell back. In this regard, we cannot exclude that the surges were not directed along the 
line of sight, but the velocities we measured were only the los components of the plasma jets ejected during the reconnection. According to this interpretation, we suggest that these reconnection processes, while quickly giving rise to the formation of filaments by trapping the cold material supplied by surges (Chae et al. 1999), might also cause their destabilization later on.

Acknowledgements. The authors wish to thank the THEMIS staff and D. Del Moro for their efficient support in the observations, as well as the team of MDI instrument on board of SOHO. This work was supported in part by the Italian Ministry of University and Research, in part by the Istituto Nazionale di Astrofisica (INAF), in part by the Agenzia Spaziale Italiana (contract I/035/0 ASI/INAF-OATO) and in part by the Università degli Studi di Catania.

\section{References}

Amari, T., Luciani, J. F., Aly, J. J., Mikic, Z., \& Linker, J. 2003, ApJ, 585, 1073 Canfield, R. C., Reardon, K. P., Leka, K. D., et al. 1996, ApJ, 464, 1016 Cavallini, F. 1998, A\&A, 128, 589

Chae, J., Qiu, J., Wang, H., \& Goode, P. R. 1999, ApJ, 513, L75

Chae, J., Qiu, J., Wang, H., \& Goode, P. R. 2001, ApJ, 584, 1084
Chae, J. 2003, ApJ, 584, 1084

Contarino, L., Romano, P., Yurchyshyn, V. B., \& Zuccarello, F. 2003, Sol. Phys., 216, 173

Kim, J., Yun H. S., Lee, S., et al. 2001, ApJ, 547, L85

Kurokawa, H., \& Kawai, G. 1993, ASP Conf. Ser., 46, 507

Lin, Y., Engvold, O., Rouppe van der Voort, L., et al. 2005, Sol. Phys., 226, 239

Malherbe, J. M. 1989, in Dynamics and Structure of Quiescent Solar Prominences (Dordrecht: Kluwer Academic Publishers), 115

Martens, P. C. H., \& Zwann, C. 2001, A\&A, 558, 872

Martin, S. F. 1998, Sol. Phys., 182, 107

Scherrer, P. H., Bogart, R. S., Bush, R. I., et al. MDI Engineering Team 1995, Sol. Phys., 162, 129

Schmieder, B., Golub, L., \& Antiochos, S. K. 1994, ApJ, 425, 326

Schwartz, P., Heinzel, P., Anzer, U., \& Schmieder, B. 2004, A\&A, 421, 323

Spadaro, D., Lanzafame, A. C., Consoli, L., et al. 2000, A\&A, 359, 716

Svestka, Z. 1976, Solar Flares (Dordrecht: Reidel)

Tandberg-Hanssen, E. 1977, in Illustrated Glossary for Solar and SolarTerrestrial Physics, ed. A. Bruzek, \& C. J. Durrant (Dordrecht: Reidel), 106 Tandberg-Hanssen, E. 1995, The Nature of Solar Prominences (Kluwer)

Wang, J., Shi, Z., \& Martin, S. 1996, A\&A, 316, 201

Welsch, B. T., \& Longcope, D. 2003, ApJ, 588

Yokoyama, T., \& Shibata, K. 1996, PASJ, 48, 353

Zhang, J., Wang, J., \& Liu, Y. 2000, A\&A, 361, 759

Zuccarello, F., Battiato, V., Contarino, L., et al. 2005, A\&A, 442, 661 